\title{
PROPOSAL OF AN AUTONOMOUS SYSTEM FOR INSPECTION OF STRUCTURES
}

\author{
Arianne Dórea Oliveira \\ Federal University of ABC \\ Santo André, São Paulo, Brazil \\ Arthur Rizzi Gama da Silva \\ FATEC Santo André \\ Santo André, São Paulo, Brazil
}

\author{
Gabriel Silva \\ Federal University of ABC \\ Santo André, São Paulo, Brazil \\ Romulo Gonçalves Lins \\ Federal University of ABC \\ Santo André, São Paulo, Brazil
}

\begin{abstract}
Inspection of defects in civil infrastructure has been a constant field of research. The enlargement of a crack, along the time, can increase the deterioration of the structure, which can result in slight problems on the material's surface until, in the serious case, the rupture of the concrete structure. In the current operational paradigm, a technician is responsible to go physically to the field in order to measure cracks in the structures. However, in terms of efficiency, the manual inspection presents many problems, such as the low accuracy of measurements taken in the field and problems for accessing high-rises, narrows places, nuclear plants, among others. Hence, the current project developed at the Federal University of ABC aims to develop a fully autonomous system capable to automate the crack measurement detection and measurement process. The proposed system uses a set of robots capable to navigate and process data by itself, ground and aerial platforms, machine vision algorithms embedded into a processing device and a remote station in order to manage all the tasks to be done. In the current proposal, the ground platform adopted is the Turtlebot $2 \AA$, which uses an embedded computer to process the programs. The aerial platform to be adopted is an eight-engine drone - octocopter that will make use of the flight controller Pixhawk 2.1 @. Both of them will be integrated and controlled from the network interconnection of several programs, such as mapping, navigation, and image processing programs. Thus, simulations will be performed by using the Gazebo $₫$ program and the Robot Operating System $\mathbb{R}$, wherein the system will be exposed to the real situations to evaluate the system efficiency. As result, once the autonomous system detects cracks, the embedded vision system algorithm must be able to process the image and assess the type and the damage caused to the inspected structure.
\end{abstract}

Keywords: structure maintenance, crack inspection, control systems, robots and automation, autonomous system inspection.

\section{INTRODUCTION}

The increase in the demand of civil constructions for diverse purposes allowed the technological development in the area of structural engineering, making studies and development of tools of detection of anomalies, ampler. Among the possible anomalies in this area are the cracks, which are pathological evidence of damage to a structure. It can be of minor gravity as is the case of cracks, average (cracks), or of more critical degree with the deep and prominent opening, classified as cracks [1].

Because of their severity, cracks require immediate attention and to do so, they must be promptly detected and as efficiently as possible, since this type of damage directly affects the durability and structural safety of the building and can cause catastrophes, for example, the Minneapolis (USA) bridge collapse in 2007 and the collapse of the Savar building in Bangladesh 2013, noticing the importance of performing periodic inspection and maintenance.

For inspections to be performed with the highest level of reliability, the use of an autonomous robotic system and the development of embedded processing technologies enable such tasks to be performed without human interference. Thus, the purpose of this work is to briefly discuss the use of such systems, to propose their architecture in a completely autonomous way for inspection of structures (aerial platform, terrestrial platform and embedded vision system for detection and measurement of cracks) and validation of their technical feasibility by performing simulations. Such systems will be integrated through the Robot Operating System ${ }^{\circledR}$, whose function is to integrate such technologies for autonomous navigation and image processing.

\section{SYSTEM ARCHITECTURE}

Autonomous systems and inspection software integrate each other via ROS, which can be understood as a distributed system, where a certain number of nodes communicate through messages. Each node of the network can 
operate in the client/server mode, which allows direct interaction between the nodes, or by publishing/signing messages, which allows the use of sensors [2]. The use of the turtlebot for mapping and navigation based on the ROS is already widespread, which offers a lot of technical support, based on bibliography available on web, as an example of the proposed use of ROS in [3] where the autonomous robot was developed for navigation and the same deviates from persons and objects, according to their respective control algorithm, and navigation of structured and unstructured external environments [4]. Moreover, besides the aforementioned examples, a robotic system assistance has been proposed and deployed in [5] and an autonomous tunnel structural inspection and assessment has been evaluated in [6].

The terrestrial platform of the proposed system uses the TurtleBot 2 robot, where the ROS has been installed in a notebook with the Linux Operating System Ubuntu 16.04 LTS, simulated in Gazebo, that receives the signals coming from the camera (for the identification and measurement of the cracks) and of the use of sensors such as Microsoft Kinect, gyroscope, accelerometer, among others, for autonomous navigation. In the aerial platform, the ROS will exchange data with the Pixhawk flight controller, where the navigation route will be pre-established so the drone can perform the inspection and measurement of the structures that according to Anac's standard must be connected to a control with a pilot case the stand-alone system fails [3]. Both the terrestrial platform and the aerial platform are being developed and validated in the Gazebo simulation platform, and the navigation programs written in $\mathrm{C}++$ and Python. Fig. 1 shows schematically the proposed architecture.

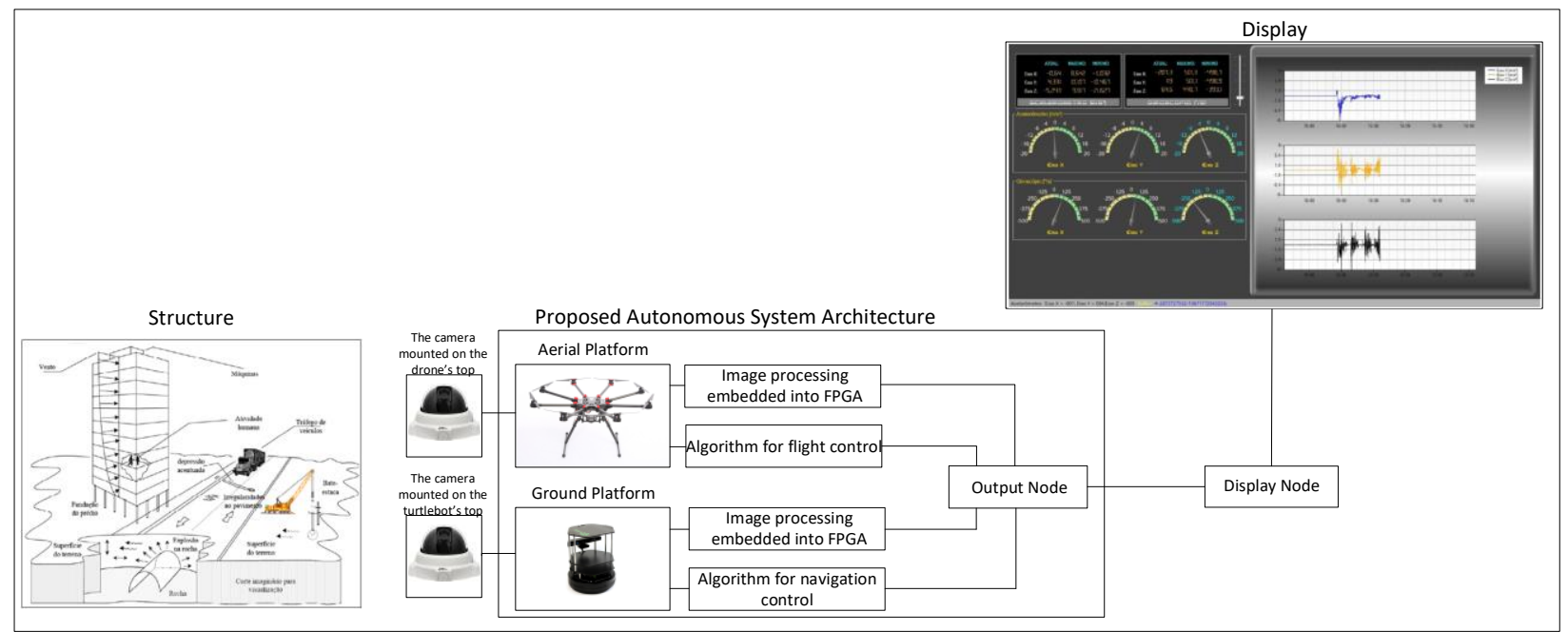

Figure 1 - Proposed Architecture of an autonomous system for inspection.

From the need to inspect a structure to be monitored, the turtlebot should be used to navigate the structure interior, such as corridors and rooms, as well as places where it can be accessed and the drone on the outside, where it is impossible to access the terrestrial platform, receiving information from their respective cameras, and both platforms will have their planning implemented by ROS. The turtlebot will have an algorithm for processing images embedded in a Field Programmable Gate Array (FPGA) board, and an algorithm of control and navigation embedded in its processing system. The drone follows the same principle of the terrestrial platform, that is, the processing of the images will be performed separately from the flight control system. The results of the image processing should be sent to the output display, as well as the flight data and trajectory data of the terrestrial platform.

\section{PRELIMINARY TESTS FOR VALIDATION}

In order to test and validate the proposed architecture, initially the platforms will be programmed and tested using a virtual environment whose the goal is to reproduce, as accurate as possible, the real environment to be inspected. Thus, the terrestrial platform is inserted into gazebo simulator, where the parameters for the simulation should be inserted and their descriptions are available in [7]. With the initial setup performed, the control algorithms are written in Python, the robot performs the autonomous navigation in the environment that simulates the floor of a building. Autonomous navigation is accomplished through the acquisition of the signals coming from the various sensors installed in the robot, such as laser scan, gyroscope, and accelerometer, among others. These signals are transformed into a point cloud, which allows the robot to identify objects, measure distance and deflect autonomously, when necessary. Fig. 2 (a) shows autonomous robot navigation through the environment. 


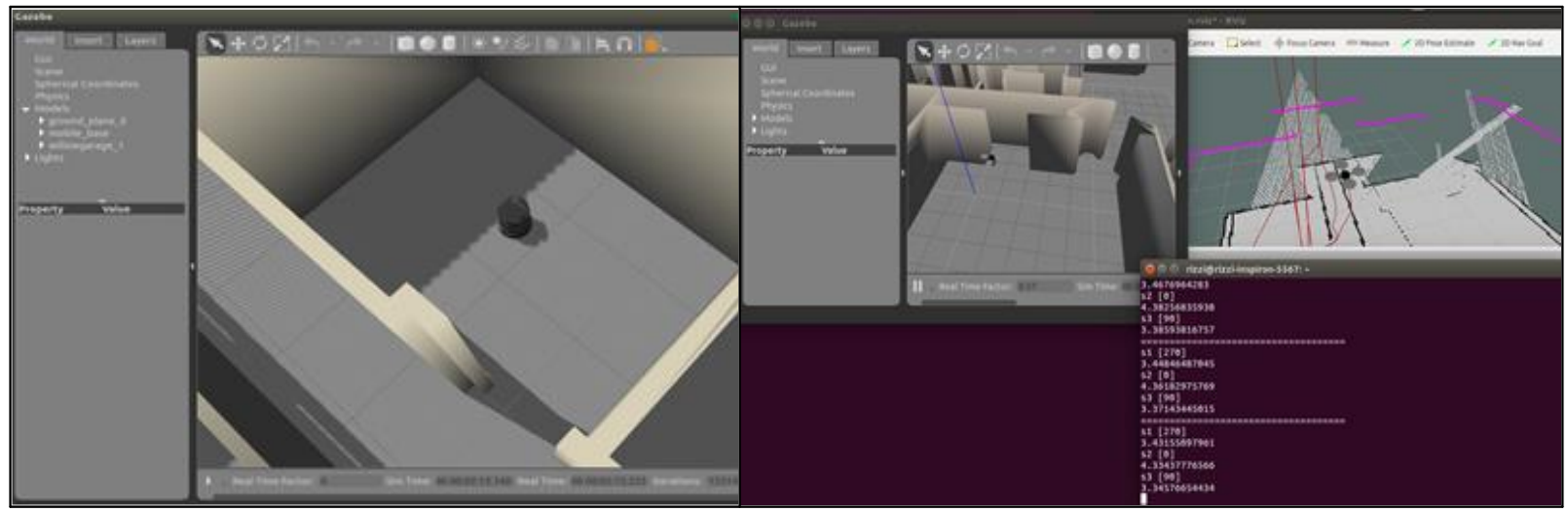

(A)

(B)

Figure 2 - Simulation of turtlebot navigating in a floor (a). Autonomous drone flight using ROS (b).

While Fig.3 illustrates the nodes and algorithms embedded in the robot's controller for ensuring the proper navigation in the internal environment.

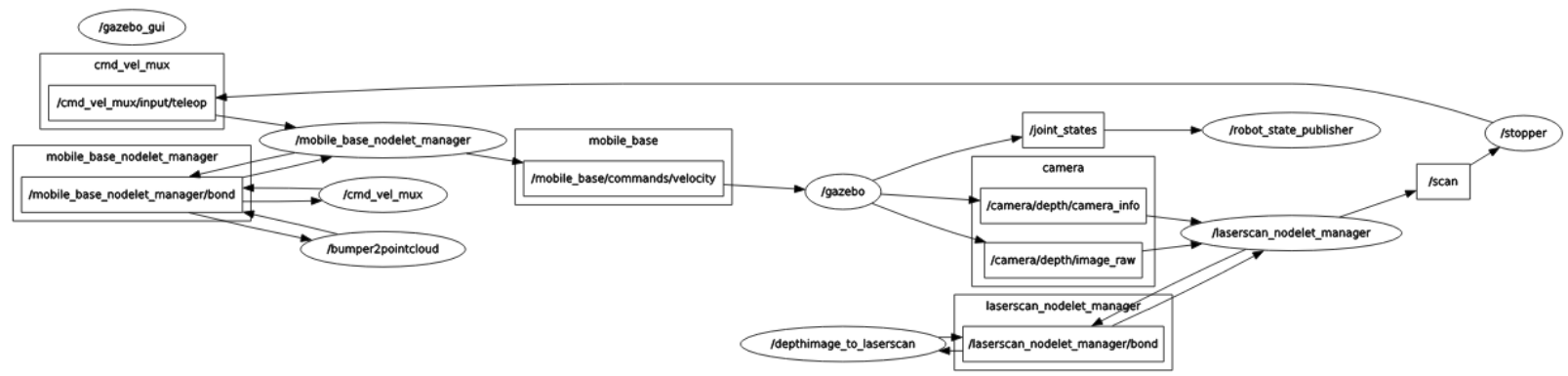

Figure 3 - ROS nodes that are currently running, as well as the ROS topics connections.

Likewise, for the ground platform, the drone has a set of sensors that allow it to fly autonomously. The hector quadrotor simulator has been used as simulator programming. First, a drone is positioned in an external environment for the initial simulations, whose the goal is to test the many functions of ROS, as well as to test the drone's mission planner. By initializing the mission, the drone starts the mission and its flight controller uses the sensors signals as inputs and updates the motors in order to accomplish the path previously established. Hence, the drone is able to navigate through the proposed environment while the camera mounted on its top takes pictures in a certain frequency. It must be noticed that the ground platform has a similar camera taking images in order to detect and measure possible cracks on surfaces. Fig. 2 (b) shows the drone flying autonomously as established previously on the mission planner.

Finally, the last element that composes the proposed architecture is the vision system for detection and measuring the cracks. According to the proposed methodology to do so, a sequence of images is processed by the crack detection algorithm in order to detect the cracks. The algorithm receives images as inputs and outputs a new image with red particles along the detected crack. The pixel positions of the particles are stored in a vector that is passed along to the crack measurement algorithm. With these pixel positions, the algorithm estimates the number of pixels in a cross section and outputs the crack dimension. It must be noticed that the aforementioned algorithm has been proposed and developed by the paper author and it is already published in [8], where accordingly to the deployed methodology, the crack detection algorithm processes a sequence of images, process all of them and outputs the pixel positions of each crack recognized. From these pixel positions, the algorithm estimates the number of pixels in each cross section and estimates the crack dimension. The statistical analysis demonstrates that the error is small on average permitting the accurate measurement of structures.

\section{CONCLUSION}

Failures in concrete structures occur at various levels of gravity and are caused by several factors. Because they are very common and due to their potential consequences, it is evident the need for new technologies that can perform inspections in the buildings in an autonomous way and in order to guarantee a result with greater fiduciary, these being 
the basis of a planning of interventions and maintenance. By using an embedded computer vision system performing autonomous inspection in such structures to detect cracks, both on a land platform and on an aerial platform, is an important technology to be developed that will have a positive impact on the area as it provides greater security to all who may make use of constructions such as bridges, buildings, or any structure which has concrete in its main component and is therefore subject to deterioration.

The tests and simulations already carried out demonstrate the understanding of the operating system that makes the interconnection of the proposed architecture and the first results about the development of the autonomous navigation indicate the current degree of autonomy of the drone and Turtlebot in the accomplishment of inspection missions.

\section{ACKNOWLEDGEMENTS}

The authors are grateful for the CNPQ support in the development of this research (process number: 408017/2016-3).

\section{REFERENCES}

[1] A. F. LOTTERMANN, " Patologias em estruturas de concreto: estudo de caso," Universidade Regional do Noroeste do Estado do Rio Grande do Sul , Ijuí, 2014.

[2] M. e. a. QUIGLEY, "ROS: an open-source Robot Operating System, p. 5.," em ICRA workshop on open source software, Kobe, 2009.

[3] T. F. CARRERA, Movimentos reativos no robô turtlebot utilizando o kinect, Bragança: Tese de Doutorado do Instituto Politécnico de Bragança, 2013.

[4] R. L. KLASER, Navegação de veículos autônomos em ambientes externos não estruturados baseada em visão computacional, São Paulo: Tese de Doutorado da Universidade de São Paulo, 2014.

[5] M. e. a. WEBSTER, "Toward reliable autonomous robotic assistants through formal verification: a case study," IEEE Transactions on Human-Machine Systems, vol. v. 46, n. n. 2, pp. p. 186-196, 2016.

[6] E. e. a. MENENDEZ, "Tunnel structural inspection and assessment using an autonomous robotic system," Automation in Construction, vol. v. 87, pp. p. 117-126, 2018.

[7] C. ROBERT, "First insights into testing autonomous robot in virtual worlds," em Software Reliability Engineering Workshops (ISSREW), 2017 IEEE International Symposium on. IEEE, p. 112-115, Toulouse, 2017.

[8] R. G. LINS e S. N. GIVIGI, "Automatic crack detection and measurement based on image analysis," IEEE Transactions on Instrumentation and Measurement, pp. v. 65, n. 3, p. 583-590, 2016. 